\title{
A trajetória da produção científica do Curso de Especialização em Gerenciamento de Serviços de Enfermagem da UNIFESP*
}

\author{
Scientific production trajectory of the São Paulo Federal University Post-Graduate \\ Program in Nursing Services Management
}

La trayectoria de la producción cientifica del Programa de Post Grado en Gerenciamento de

Servicios de Enfermería de la Universidad Federal de São Paulo

\author{
Camila Kelly Gomes de Lima', Maria Cristina Sanna' \\ 'Universidade Federal de São Paulo, Departamento de Enfermagem, Grupo de Estudos e Pesquisa em \\ Gerenciamento dos Serviços de Saúde e Enfermagem. São Paulo, SP
}

Submissão: 10/05/2007

Aprovação: 17/04/2008

\section{RESUMO}

Este artigo descreve a trajetória da produção científica do Curso de Gerenciamento de Serviços de Enfermagem da UNIFESP a partir da análise das monografias produzidas pelos alunos nos dez anos de existência do curso. Com o objetivo de Identificar temas e objetivos e desenhar linhas de peseuisa a Que corresponderam esses trabalhos, 113 monografias foram estudadas. A apuração de fręüência simples e relativa das variáveis estudadas e a classificação da temática segundo referencial teórico sobre o assunto indicou Que, no início, os estudos não se atinham à área de abrangência do curso, mas paulatinamente foram se orientando para esta, com destaque para "Recursos Humanos", o que se acelerou com a criação de grupo de pes@uisa focado na sub-especialidade "Gerenciamento em Enfermagem".

Descritores: Enfermagem; Gerência; História da enfermagem; PesQuisa em enfermagem.

\begin{abstract}
This article describes the scientific production trajectory of the Post-Graduate Program in Nursing Services Mangement at São Paulo Federal University based on the analysis of monographies produced by the students over the last 10 years. The study aimed at identifying subjects and objectives and to delineate research lines of these studies, 113 monographs were analyzed. The verification of the simple and relative freeuency of the studied variables and the thematic classification according to the theoretical referential on this subject showed that at the beginning the studies did not considered the area of course extent but gradually they began to reach it with emphasis in "Human Resources", which was accelerated with the establishment of a research group with the focus in the subspecialty "nursing management".
\end{abstract}

Descriptors: Nursing; Managment; History of nursing, Nursing research.

\section{RESUMEN}

Este artículo describe la trayectoria de la producción científica del curso de Administración de Servicios de Enfermería de la UNIFESP a partir del análisis de las monografías producidas por los alumnos en los diez años de existencia del curso. Con el objetivo de identificar los temas y objetivos y delinear líneas de pesquisa referentes a esos trabajos, 113 monografías fueron estudiadas. La apuración de frecuencia simple y relativa de las variables estudiadas y la clasificación de temática según el referencial teórico sobre el asunto indicó Que, al inicio, los estudios no se restringieron al área de abarcamiento del curso pero paulatinamente, fueron se orientando hacia esta, con destaque para "Recursos Humanos", lo Que se aceleró con la institución del grupo de pesquisa con foco en la subespecialidad "Administración en Enfermería".

Descriptores: Enfermería; Gerencia; Historia de la enfermería, Investigación en enfermería 


\section{INTRODUÇÃO}

A Pós-Graduação no Brasil, aprovada pelo Conselho Federal de Educação, em 1965, foi criada para atender à necessidade de ampliar a capacidade de investigação das universidades e de seu corpo docente. O objetivo dessa medida foi a formação de professores competentes que pudessem atender à expansão Quantitativa do ensino superior, estimular o desenvolvimento da pesquisa por meio de preparação de novos pesquisadores e assegurar o treinamento eficaz de técnicos e trabalhadores intelectuais do mais alto nível, para fazer face às necessidades do desenvolvimento em todos os setores ${ }^{(1)}$. A partir de então, com a implantação dos cursos de pósgraduação stricto sensu - Mestrado e Doutorado ${ }^{(2)}$, com início em 1972, a pesquisa em Enfermagem passou a ser mais valorizada no nosso país.

O conhecimento na área de Enfermagem se distribui hoje em diversas linhas de pesquisa, Que são definidas por Sanna ${ }^{(3)}$ como o agrupamento de uma produção científica por afinidade temática e/ ou metodológica. A construção de uma linha de pesquisa decorre da atividade de um determinado grupamento de estudiosos Que, geralmente sob a liderança de um pesquisador mais experiente, acaba por produzir e submeter à avaliação pelos pares, Quantidade suficiente de relatórios de peseuisa com a afinidade acima descrita. A classificação das linhas de pesquisa em Enfermagem é assunto controverso e desperta grande mobilização dos enfermeiros ${ }^{(4,5)}$.

A produção do conhecimento sobre Gerenciamento em Enfermagem no Brasil tem sido objeto de alguns estudos( ${ }^{(6,7)} \mathrm{e}$ discussões no circuito dos Programas de Pós-Graduação, o que tem subsidiado importantes decisões, tanto Quanto às práticas gerenciais e de ensino, como da pesquisa sobre o tema. Já sobre a pós-graduação senso estrito, de maneira geral, pouco se tem pesquisado, havendo esparsas publicações ${ }^{(8-1)}$ sobre a produção científica Que congregam e o impacto Que causam.

A pós-graduação lato sensu, Que era oferecida desde a década de 1940, na Escola Anna Nery ${ }^{(9)}$, é definida como um instrumento de capacitação de enfermeiros e é voltada para áreas técnicocientifico especificas da Enfermagem ${ }^{(8)}$, principalmente no âmbito da assistência, mas não só nela encerrada. Fazem parte dessa modalidade de ensino os cursos de atualização, aperfeiçoamento e especialização, dentre os Quais figura o de Gerenciamento em Enfermagem.

O Departamento de Enfermagem da Universidade Federal de São Paulo (UNIFESP), vem oferecendo regularmente cursos de especialização em Enfermagem desde 1972, em acordo com a legislação vigente, contribuindo, assim, para o desenvolvimento profissional da Enfermagem no país ${ }^{(8)}$. Dentre esses se destaca o Curso de Especialização em Gerenciamento de Serviços de Enfermagem, Que foi ministrado pela primeira vez em 1993, com 418 horas, oferecendo um tronco comum junto às Especializações em Enfermagem do Trabalho e Enfermagem em Saúde Publica.

O curso foi ampliado e reestruturado, passando a ser oferecido, a partir de 1996, com carga horária de 900 horas ${ }^{(8)}$. Atualmente, tem carga horária de 504 horas e foi idealizado visando proporcionar, ao enfermeiro, a oportunidade de ampliar e aprofundar o conhecimento sobre teorias, princípios e finalidades da administração e organização de serviços, e o gerenciamento da estrutura, do processo e dos resultados do cuidar em Saúde e
Enfermagem. O curso contempla disciplinas e monografia como desenho regular desde o seu início.

Considerando Que o Departamento de Enfermagem vem oferecendo esse curso há uma década, faz se necessário investir no aprofundamento do conhecimento sobre sua história. Sabe-se que o curso já especializou mais de uma centena de enfermeiros, inclusive de outros Estados do país, Que desenvolveram trabalhos de pesQuisa nas instituições de origem, resultando em monografias voltadas para a melhoria da prática de enfermagem nos serviços de saúde ${ }^{(4)}$, mas ainda não é conhecida a extensão e especificidade dessa produção científica.

Chama à atenção o fato deste curso ser referência nessa área por estar vinculado a instituição de grande prestígio, com significativa presença no cenário da pesquisa no país e mesmo fora dele. Dessa forma, o que se faz nele ajuda a compreender o que se faz em pesQuisa em Administração em Enfermagem. Nesse contexto, tornase necessário identificar as áreas de conhecimento às Quais as monografias produzidas durante os dez anos de existência do curso se vinculam, analisar os elementos Que influenciaram na seleção do referencial teórico e metodológico e identificar as linhas de pesquisa Que foram desenvolvidas nesse período.

O estudo oferecerá uma visão pontual mas pertinente da produção científica desenvolvida pela pós-graduação latu sensu em Gerenciamento de Serviços de Enfermagem da UNIFESP, numa visão retrospectiva Quanto às áreas e linhas de pesquisa praticadas durante a existência do curso.

\section{OBJETIVOS}

- Geral: descrever a trajetória da produção cientifica do Curso de Especialização em Gerenciamento em Enfermagem da UNIFESP.

- Específicos: Caracterizar a situação dos alunos no curso e a distribuição de sua produção monográfica por tema e ano de apresentação; e Desenhar as linhas de pesquisa a Que corresponderam esses trabalhos, comparando-as com referencial teórico sobre o assunto, a partir da identificação de temas e objetivos declarados pelos alunos que concluíram o curso em suas monografias.

\section{METODOLOGIA}

Estudo d3escritivo-explorat 'roio, de caráter histórico, cujas fontes de pesquisa foram registros acadêmicos e cópias das monografias dos alunos concluintes.

Para realização da presente investigação, inicialmente realizouse um contato com a coordenadora do curso de Especialização em Gerenciamento dos Serviços de Enfermagem da UNIFESP, para obtenção de autorização de acesso ao arQuivo das monografias impressas ou armazenadas em disquetes entregues pelos alunos, Que ficava na Secretaria do curso.

Em seguida, para a caracterização da produção dos alunos, foram solicitadas e obtidas cópias dos seguintes documentos: lista de todos os alunos matriculados, lista de todos os alunos concluintes e lista dos temas das monografias e respectivos orientadores.

Concomitante à coleta das informações nos documentos citados, organizou-se a construção de um banco de dados em planilha Excel com as seguintes variáveis: ano de apresentação, nome do aluno, 
nome do orientador e título do trabalho. Por meio desse instrumento foi possível calcular a freeüência simples e relativa das variáveis selecionadas para o estudo.

$\mathrm{O}$ acesso aos documentos foi um trabalho extenuante por causa da dificuldade do manejo para coleta de informações para a pesQuisa, visto Que os mesmos não estavam organizados, separados por turma e por ano, sendo necessários vários dias de buscas e outros tantos para identificação e anotação das informações desejadas. Durante esse percurso, um fato curioso foi o encontro, entre os documentos eletrônicos pesquisados, de Quatro disquetes com arquivos pessoais dos alunos e 12 monografias de outros cursos. Também não foi possível acessar os arquivos contidos em cinco disquetes, por falha intrínseca do dispositivo eletrônico.

A tentativa de classificar os trabalhos conforme os resumos, Que estava prevista na segunda etapa do desenho da pesquisa, foi prejudicada porque este não constava da maioria dos textos obtidos. Assim, trabalhou-se com as monografias na íntegra, enfocando-se o título e o objetivo com prioridade, para a identificação das linhas de pesquisa seguidas pelos autores. Esses títulos foram classificados segundo referencial teórico específico para a ordenação do conhecimento sobre Administração em Enfermagem ${ }^{(12)}$, nos seguintes itens: Bases Ideológicas e Teóricas da Administração em Enfermagem; Processos de Intervenção - Planejamento, tomada de decisão, Auditoria e Supervisão; Gerenciamento de Recursos Físicos; Gerenciamento de Recursos Materiais; Gerenciamento de Recursos Humanos; Gerenciamento de Recursos Financeiros; Gerenciamento de Recursos de Informação e Gerenciamento de Recursos Políticos. Sua análise foi processada em função da concentração e distribuição da temática no tempo, com o Quê foi possível descrever a trajetória percorrida no período estudado.

Acrescente-se ainda Que, apesar de não se tratar de pesquisa com seres humanos, por exigência institucional, o projeto foi submetido ao Comitê de Ética em Pesquisa da Universidade Federal de São Paulo e aprovado.

\section{RESULTADOS E DISCUSSÃO}

A caracterização da situação dos alunos do curso em agosto de 2006 pode ser apreciada na Tabela 1. Nela está evidenciado Que, de 1996 a 2005, o curso teve 278 alunos matriculados, com exceção do ano de 1997, Quando se apurou Que o curso não foi oferecido. Dos matriculados, 125 (44,96\%) já concluíram o curso, $16(5,75 \%)$ foram reprovados, I I $(3,96 \%)$ trancaram a matrícula e 75 (45,33\%) ainda o estão cursando.

Verificou-se Que a reprovação e o trancamento da matrícula são fenômenos menos freeüentes. Já os alunos formados estão na mesma proporção dos alunos Que continuam cursando, o Que é surpreendente, pois se encontrou alunos da primeira turma Que, após uma década, são considerados como ainda vinculados regularmente ao curso. A surpresa decorre da percepção de Que o grande avanço tecnológico tende a tornar o conhecimento científico adeuirido no curso insuficiente ou obsoleto após um período de tempo, a ponto de ser necessário cursar as disciplinas novamente para obtenção do título de especialista em gerenciamento de serviços de enfermagem.

Outro achado importante foi a oscilação do número de formados ao longo do tempo, visto Que há anos, como em 2000, em Que se chegou à aprovação de até $79 \%$ do total matriculado e, em 2004 , por exemplo, só $32 \%$ concluiu o curso. Foi notado também Que, em 2005 - um ano antes da realização da peseuisa, nenhum aluno havia concluído o curso, e Que a concentração dos Que continuam matriculados Que já podem ter encerrado os créditos em disciplinas se avoluma no último lustro, o Que sugere Que raramente o aluno consegue obter o título ao final de um ano de curso, como previsto no programa.

A explicação para o tempo estendido para a conclusão do curso pode estar relacionada à elaboração e entrega da monografia. Isto enfatiza a importância atribuída pelos organizadores do curso para esta etapa de formação do especialista, e sinaliza Que o desenvolvimento desta atividade merece atenção especial.

De autoria dos 125 alunos Que concluíram o curso, foram encontradas 113 monografias, sendo elas de todos os anos em Que o mesmo foi oferecido, registrando-se uma perda de $0,9 \%$. Destes 113 trabalhos analisados, foram excluídas 34 monografias por não se referirem ao objeto de estudo. .Elas estavam assim distribuídas: 25 eram da área assistencial, Quatro versavam sobre caracterização de clientela, uma tratava de tema abrangente sobre administração em saúde, e as outras Quatro não eram relatórios de pesQuisa e sim relatos de experiência (uma), projetos de intervenção na realidade de trabalho (duas) e uma proposta teórica de organização de um serviço assistencial.

O total de monografias analisadas ficou, então, em 79, ou 69,91\% do grupamento de textos encontrados e 63,25 do total de concluintes do curso no período estudado, respectivamente. A distribuição temática do conjunto pode ser apreciada na Tabela 2 .

Verificou-se Que a grande concentração das monografias está localizada em duas áreas temáticas: Gerenciamento de Recursos Humanos, com 29 títulos, Que correspondem a 37\% do total, seguida de Métodos de Intervenção, com outros 22 títulos, correspondendo a $28 \%$ do total. Essa concentração justifica-se, em parte, pela problemática mais contundente vivida pelos enfermeiros no cotidiano de trabalho ser a relacionada ao gerenciamento daqueles recursos e, por outra parte, pelo fato da produção científica em Enfermagem no Brasil, até a década de 1990, não dedicar ênfase aos métodos

Tabela I. Situação dos alunos no segundo semestre de 2006.

\begin{tabular}{lcccccccccc}
\hline Ano/Situação & 1996 & $\mathbf{1 9 9 8}$ & $\mathbf{1 9 9 9}$ & $\mathbf{2 0 0 0}$ & $\mathbf{2 0 0 1}$ & $\mathbf{2 0 0 2}$ & $\mathbf{2 0 0 3}$ & $\mathbf{2 0 0 4}$ & 2005 & Total \\
\hline Formados & 9 & 4 & 13 & 22 & 22 & 21 & 19 & 15 & - & 125 \\
Trancados & - & 1 & - & - & - & 1 & 9 & - & - & 11 \\
Reprovados & - & - & 4 & 4 & - & 5 & - & 3 & - & 16 \\
Cursando & 12 & 1 & 4 & 2 & 13 & 14 & - & 29 & 51 & 75 \\
\hline Total & 21 & $\mathbf{6}$ & 21 & $\mathbf{2 8}$ & $\mathbf{2 5}$ & $\mathbf{4 1}$ & $\mathbf{2 8}$ & $\mathbf{4 7}$ & $\mathbf{5 1}$ & 278 \\
\hline
\end{tabular}


Tabela 2. Monografias produzidas no período de 1996 a 2005, categorizadas segundo Sanna ${ }^{(7)}$.

\begin{tabular}{lcc}
\hline Temas & N & \% \\
\hline Bases Ideológicas e Teóricas & 1 & 1,27 \\
Métodos de Intervenção & 22 & 27,84 \\
Recursos Humanos & 29 & 36,71 \\
Recursos Materiais & 1 & 1,27 \\
Recursos Físicos & - & - \\
Recursos Financeiros & 4 & 5,06 \\
Recursos Políticos & 12 & 15,19 \\
Recursos de Informação & 10 & 12,66 \\
\hline Total & 79 & 100 \\
\hline
\end{tabular}

Tabela 3. Monografias sobre Gerenciamento de Recursos Humanos.

\begin{tabular}{lcc}
\hline Temas & N & $\%$ \\
\hline Dimensionamento & 7 & 24,14 \\
Recrutamento & - & - \\
Seleção & - & - \\
Distribuição & - & - \\
Avaliação de desempenho & 6 & 20,67 \\
Educação Continuada & 9 & 31,03 \\
Saúde ocupacional & 6 & 20,67 \\
Formação & 1 & 3,49 \\
\hline Total & 29 & 100 \\
\hline
\end{tabular}

empregados na administração dos serviços de enfermagem.

Esses dados corroboram com a análise feita num estudo ${ }^{8}$ Que identificou o mesmo predomínio de pesquisas sobre Gerenciamento de Recursos Humanos, com 30\% do total - o tema mais estudado nas décadas de 1970 a 2000, em outro centro de investigação também localizado na cidade de São Paulo.

Conforme ilustra a Tabela 3, as 29 monografias agrupadas na grande área de Gerenciamento de Recursos Humanos foram classificadas em: Educação Continuada $31 \%$, Dimensionamento 24\%, Saúde Ocupacional $21 \%$, Avaliação de Desempenho $21 \%$ e Formação 3\%. Não foi encontrada nenhuma monografia Que abordasse Recrutamento, Seleção e Distribuição de pessoal. A área de Recursos Humanos também foi a única Que esteve presente em todos os anos em Que o curso foi oferecido, e sempre com boa representatividade, o Que permite afirmar Que esta temática é muito valorizada na UNIFESP.

Sobre Métodos de Intervenção, Auditoria, com 86\%, concentrou o maior número de trabalhos, seguida de Planejamento com 9\%, finalizando com Tomada de Decisão com 5\%. Foi possível verificar Que nenhum estudo abordou a Supervisão, o que é um fato inesperado para um curso de Gerenciamento. O tema Auditoria foi o Que apresentou mais monografias na trajetória do curso, e a multiplicação de trabalhos sobre esta temática vem se acentuando nos últimos anos, como se pode apurar.

O Gerenciamento de Recursos Políticos com um total de 12 monografias teve a atenção dividida entre: Motivação com 40\% dos títulos, Liderança com 27\%, Relacionamento Interdepartamental com $27 \%$, e Poder e Cultura com $6 \%$.

lá sobre Recursos de Informação foram encontrados dez trabalhos, assim distribuídos: Prontuários 6, Informática 2, Impressos I e Comunicação I.
Como a Tabela 2 indica, é escassa a produção sobre Gerenciamento de Recursos Financeiros (5\%), Gerenciamento de Recursos Materiais (1\%) e Bases Ideológicas e Teóricas da Administração em Enfermagem (1). Nenhuma monografia abordou o Gerenciamento de Recursos Físicos. O aparente pouco interesse pelos últimos temas mencionados aponta para eventuais lacunas na produção do conhecimento nessas áreas.

Percebeu-se Que com a progressiva aQuisição de maturidade pelo curso, foram se construindo as linhas de peseuisa do grupo Que se responsabiliza por sua produção. Nesse cenário, a área de Recursos Humanos é significativa e a gradativa orientação da produção científica do curso para linhas de pesQuisa vinculadas à área de abrangência do mesmo acaba por fortalecer esse grupo de pesquisadores, Que podem evoluir para formalização de grupos, como ocorre em outros centros de produção do conhecimento.

De fato, em 2003 foi criado O GEPAG - Grupo de Estudos e Pesquisas em Administração de Serviços de Saúde e Gerenciamento de Enfermagem, Que possui atualmente Quatro linhas de pesquisa Que são: Formação e Capacitação de Recursos Humanos em Saúde e Enfermagem, Fundamentos e Práticas de Gerenciamento de Serviços de Saúde e de Enfermagem, História do Gerenciamento e Administração em Saúde e Enfermagem e, por fim, Programas de Melhoria de Qualidade e Avaliação das Práticas de Enfermagem.

Como desdobramento da produção científica e desenvolvimento da pesQuisa no curso em foco e, também na pós-graduação sensu stricto, em 2006 foi criado o GEPAV-SE - Grupo de Estudos e Pesquisa em Avaliação de Qualidade em Serviços de Saúde e Enfermagem, originado do GEPAG. A formalização do grupo demonstra Que a produção nessa área está crescendo e Que a expressividade numérica detectada no presente trabalho, no que 
se refere à Auditoria, foi bastante para sustentar a criação deste segundo grupo.

Observou-se que os pesquisadores da instituição, sejam alunos, ou professores Que se dedicam à área de Gerenciamento em Enfermagem, deflagraram uma produção significativa nesse campo, no Qual, o curso de especialização ocupa papel de destaque porQue é o Que mais títulos produziu, tornando-se um canal para fechar as linhas de pesquisa, conforme evidenciado pela trajetória ora apresentada.

\section{CONCLUSÕES}

O curso de pós-graduação latu sensu de Especialização em Gerenciamento em Enfermagem da UNIFESP vem contribuindo para a Qualificação dos enfermeiros Que atuam no ensino, pesQuisa e assistência, em áreas especificas de atuação, com representativa produção científica.

Como aspecto a ser destacado na análise da trajetória dessa produção, apreendeu se Que há demora significativa para a conclusão do curso, sendo pouco freQüente a obtenção do título ao final do mesmo, o Que pode estar relacionado com a elaboração da monografia. Cerca de 50\% dos alunos matriculados nos últimos dez anos já concluíram o curso com a entrega da monografia, estando boa parte ainda na condição de matriculado e não concluinte.

Quanto aos temas das monografias, no inicio do curso, observouse Que os autores transitaram por aspectos assistenciais, o Que fez supor Que não havia orientação para seguir linhas de pesQuisa previamente definidas e vinculadas ao curso. Com o amadurecimento deste, essas monografias foram deixando de existir, concentrandose a produção mais no âmbito gerencial.

O Gerenciamento de Recursos Humanos foi a temática prevalente, estando presente em todos os anos em Que o curso foi oferecido, seguido de Auditoria, mais freqüente nos últimos anos, caracterizando-se assim, como linhas de pesquisa mais estruturadas. Houve nenhum ou peQueno interesse por temas como Recursos Físicos, Recursos Materiais e Bases Ideológicas e Teóricas da Administração em Enfermagem, o Que indica Quais escolhas estão sendo feitas no direcionamento da pesQuisa por esse grupamento de orientadores e alunos.

Considerou-se ainda Que a criação de dois grupos de pesQuisa o GEPAG e o GEPAV-SE, ocorridas recentemente, são resultantes da produção cientifica significativa nessa área de conhecimento, na instituição em foco.

No decorrer da reconstrução da trajetória de pesQuisa do Curso de Especialização em Gerenciamento de Serviços de Enfermagem da UNIFESP foram encontrados resultados positivos. Tal positividade não significa a inexistência de problemas mas, ao contrário, aponta para alguns aspectos a serem analisados e Que, uma vez considerados, possam efetivamente contribuir para a melhoria do ensino de pósgraduação em Gerenciamento e para a organização de esforços de pesQuisa na direção de construção do conhecimento sobre Administração em Enfermagem. As fragilidades identificadas merecem desenvolvimento de projetos de investigação albergados nos respectivos grupos de peseuisa, para com isso encaminhar a tomada de decisões sobre a condução do Curso de Gerenciamento de Serviços de Enfermagem e dos grupos em que as linhas se desenvolvem.

\section{REFERÊNCIAS}

1. Almeida MCP, Rodrigues RAP, Furegato ARF. A pós-graduação na Escola de Enfermagem de Ribeirão Preto-USP: evolução histórica e sua contribuição para o desenvolvimento da enfermagem. Rev Latino-am Enfermagem 2002; 10(3): 27687.

2. Lopes GT. A trajetória da investigação científica no âmbito da enfermagem. Esc Anna Nery Rev Enferm 2002; 6(1):53-62.

3. Sanna MC. Linhas de pesquisa. [citado em: 28 jun 2006]. Disponível em: URL: http://mcsanna.sites.uol.com.br/ linhas_pesouisa.htm

4. Carvalho V. Linhas de pesquisa e prioridades de enfermagem: proposta com distinção gnosiológica para o agrupamento da produção cientifica de pós-graduação em enfermagem. Esc Anna Nery Rev Enferm 2002; 6( I): 145-54.

5. Associação Brasileira de Enfermagem. Consolidação das propostas de linhas de pesquisa em enfermagem. Brasília (DF); ABEn; 200I. [citado em 17 maio 2006]. Disponível em http:/ /www.abennacional.org.br/documentos/linhapesel I senpe.doc

6. Kurcgant P, Ciampone MHT. A pesquisa na área de Gerenciamento em Enfermagem no Brasil. Rev Bras Enferm 2005; 58(2): 16I-4.

7. Ciampone MHT, Felli VEA, Castilho V, Kurcgant P. A produção do conhecimento na área de administração de serviços de Enfermagem do programa de pós-graduação. Rev Esc Enferm USP 2005; 39(esp): 535-43.

8. Barros SMO, Ribeiro CA, Sumita SLN, Cunha ICKO, Granitoff $\mathrm{N}$, Abrahão AR, et al. Pós-graduação lato senso em Enfermagem na Universidade Federal de São Paulo. Acta Paul Enferm 1998; II (esp): 17-2 I.

9. Santos TCF, Sousa MI, Araújo STC. A pós-graduação em Enfermagem "lato senso". Esc Anna Nery Rev Enferm 1997; I (esp): 93-8.

10. Brito RS, Monteiro Al, Aguila LTM, Timóteo RPS, Enders BC, Germano RM, Oliveira FVS. Avaliação e perspectiva dos cursos de pós-graduação lato-sensu do Departamento de Enfermagem da UFRN (1980-1 992). Rev Bras Enferm 1995; 48(I): 26-32.

11. Guedes MVC, Silva LF, Freitas MC. Impacto dos cursos de especialização na transformação da prática do enfermeiro: um estudo sobre o valor da pesquisa. RENE 2000; I ( I): 36-40.

12. Sanna MC. A Estrutura do Conhecimento sobre Administração em Enfermagem. Rev Bras Enferm 2007; 60(3): 336-8. 